\title{
Sciences du démantèlement des installations nucléaires
}

Sous la direction de Robert DAUTRAY et Yves BRÉCHET

ACADÉMIE DES SCIENCES

edpsciences

17, avenue du Hoggar

Parc d'activités de Courtabœuf, BP 112

91944 Les Ulis Cedex A, France 
Illustrations de couverture : en haut à gauche : réacteur expérimental Siloé sur le centre CEA de Grenoble, (C) CEA Grenoble ; en haut, à droite : démantèlement de Siloé, ( ) P. Avavian/CEA ; en bas : Futur quartier (vue d'artiste), (c) CEA Tech.

\section{Imprimé en France}

\section{(c) 2015, EDP Sciences}

17, avenue du Hoggar, BP 112, Parc d'activités de Courtaboeuf, 91944 Les Ulis Cedex A

Tous droits de traduction, d'adaptation et de reproduction par tous procédés réservés pour tous pays. Toute reproduction ou représentation intégrale ou partielle, par quelque procédé que ce soit, des pages publiées dans le présent ouvrage, faite sans l'autorisation de l'éditeur est illicite et constitue une contrefaçon. Seules sont autorisées, d'une part, les reproductions strictement réservées à l'usage privé du copiste et non destinées à une utilisation collective, et $d$ 'autre part, les courtes citations justifiées par le caractère scientifique ou d'information de l'oeuvre dans laquelle elles sont incorporées (art. L. 122-4, L. 122-5 et L. 335-2 du Code de la propriété intellectuelle). Des photocopies payantes peuvent être réalisées avec l'accord de l'éditeur. S'adresser au : Centre français d'exploitation du droit de copie, 3, rue Hautefeuille, 75006 Paris. Tél. : 0143269535. 